\title{
Quasi-static and Fatigue Delamination at Tape/Fabric Interfaces
}

\author{
Carlos G. Dávila ${ }^{1}$ and James Ratcliffe ${ }^{1}$ \\ NASA Langley Research Center, Hampton, VA, 23692, USA
}

\begin{abstract}
The relationship between quasi-static and fatigue delamination of a fabric/tape interface is examined experimentally and numerically. Mixed-mode bending tests were conducted using specimens in which a mid-plane delamination is bound between a ply of 0-degree unidirectional tape and a 0-degree fabric ply of the same material system. The experimental results indicate that delaminations tend to migrate towards the constraining ply that is on the compressive side of the laminate in bending, and that the fracture toughness for a fabricconstrained delamination is almost twice that of a tape-constrained delamination. A cohesive model based on superposition of bilinear laws was used to account for these differences in measured properties. Fatigue analyses were conducted with a cohesive damage model that uses an idealization of stress-life diagrams used in engineering design. The fatigue model is shown to be capable of predicting the steady-state rate of delamination propagation described by the Paris law, as well as the initial transients that depend on the quasi-static R-curve effects. The analysis results help quantify the effects of fracture toughness and R-curves on the rates of delamination propagation in fatigue.
\end{abstract}

\section{Skin/Stiffener Delamination and Tape/Fabric Interfaces}

The collapse of stiffened panels is usually the result of skin/stiffener separation stemming from the presence of local damage caused by impact, manufacturing, or fatigue, or from compressive loads that induce postbuckling deformations. Typical composite fuselage skins are composed of unidirectional plies, while the stiffeners, which have tighter compound curvatures and require materials with good drapability, are often manufactured with fabric plies. The tape/fabric interface between skin and stringer along the edge of the stiffener flange is particularly susceptible to the effects of impact damage and delamination. Figure 1 illustrates a typical hat stiffened construction, where the red outer stiffener plies and the blue inner stiffener wrap correspond to fabric plies, while the gray surface represents a skin manufactured with unidirectional tape. The smaller inset figure depicts a stiffener pull-off test used to assess the ability of the subelement to sustain loads without skin/stiffener separation.

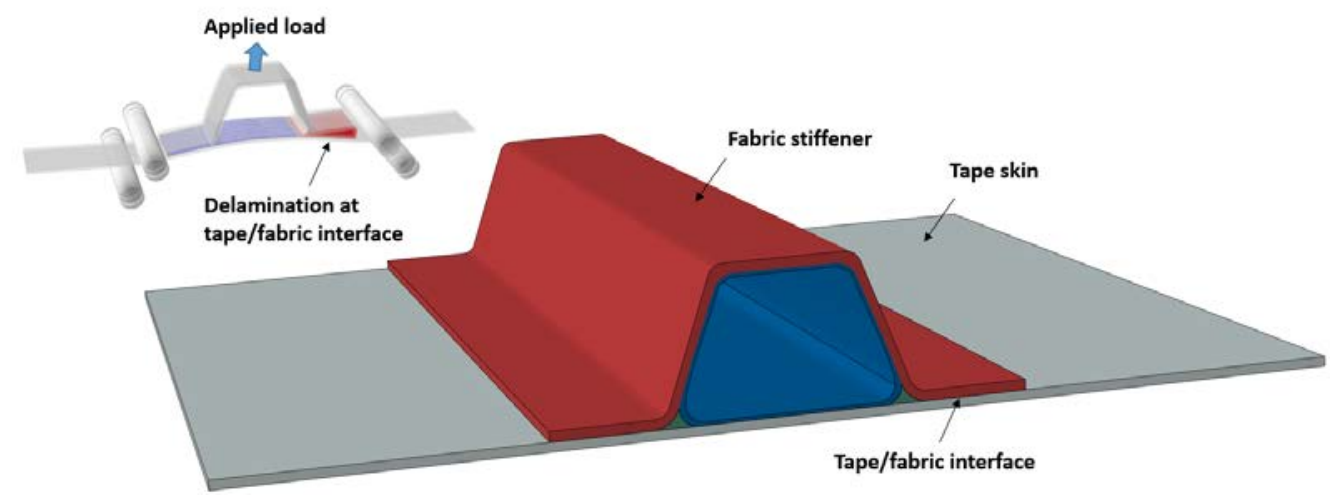

Fig. 1 Hat-stiffed panel with fabric and tape construction and hat pull-off test.

\footnotetext{
${ }^{1}$ Aerospace Research Engineer, Durability, Damage Tolerance, and Reliability Branch, AIAA Member.
} 
Recent studies have indicated that the interfaces between dissimilar materials, such as between tape and fabric, have fracture properties that can differ significantly from those of standard specimens composed of 0-degree unidirectional (UD) tape. Furthermore, it has been shown that the fracture properties of delaminations constrained at the interface between two dissimilar materials depend upon which of the two surfaces is active in constraining the path of the delamination. Based on fractographic observations of delamination surfaces, Greenhalgh [1] described the tendency of a delamination to migrate toward one or the other surface according to the direction of the resolved tensile stress, $\sigma_{\mathrm{r}}$, along the delamination front. Under mixed-mode loading, the natural tendency of the delamination front is to migrate toward the compressive face of the laminate under bending. This concept is illustrated in Figure 2 in the context of the present study on tape/fabric interfaces. A case is shown in Figure 2a where the resultant stress state, and overall direction of macroscopic delamination growth, lead to the formation of microcracks that tend towards the UD tape ply. Since the delamination cannot migrate through the fibers, the UD ply is said to contain the delamination. Figure $2 \mathrm{~b}$ corresponds to a case where the containing ply is now the fabric ply. In these examples, when the migration is towards the tape, the delamination encounters a smooth surface that is similar to that of a standard specimen. Instead, when the delamination is contained by fabric, the delamination finds a more tortuous path that can lead to rougher fracture surfaces, multiple paths through the woven plies, and a higher fracture toughness [2]. This interaction between micro-cracking and the neighboring plies is typically not considered during the characterization of interlaminar fracture.

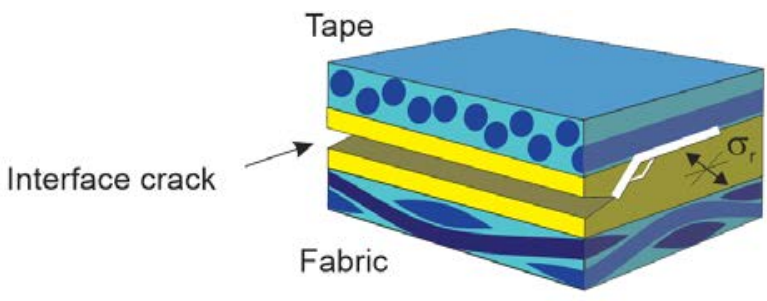

(a) Containment ply: UD tape

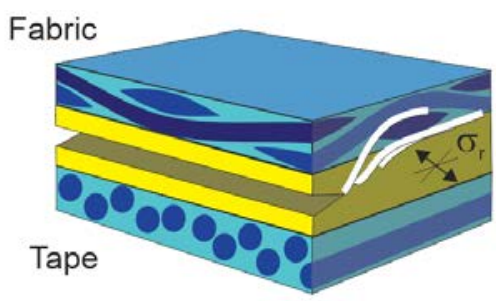

(b) Containment ply: fabric

Fig. 2 Interaction between neighboring plies and microcracks under mixed-mode loading

\section{Characterization of the Fracture Properties of Tape/fabric Interfaces}

The panels designed for the present study were manufactured with IM7/8552 UD tape and plane weave (PW) fabric. To characterize the properties of the tape/fabric interface, specimens were fabricated with a stacking sequence of $\left[0_{t}\right] /\left[0_{f}\right] /\left[0_{t}\right]_{5} /\left[0_{f}\right] /\left[0_{t}\right] / T /\left[0_{f}\right] /\left[0_{t}\right]_{7} /\left[0_{f}\right]$, where the subscript " $t$ " refers to tape, "f" refers to fabric, and $T$ stands for the crack initiator Teflon. This stacking sequence results in a laminate that is approximately symmetric and balanced, but with a fracture interface between tape and fabric. Ratcliffe describes the configuration of the specimens and the testing procedures in Refs. [2, 3].

The specimens were tested in a previous test campaign in double cantilever beam (DCB), end-loaded split (ELS) and mixed-mode bending (MMB) tests [4]. The ELS and MMB tests were conducted with specimens oriented with tapeside-up and fabric-side-up to evaluate the influence of the shear stress direction on the crack growth resistance.

The experimental results indicate that, in all tests, the fracture plane remains at the tape/fabric interface and does not migrate. Typical experimental force-displacement results for a DCB and an MMB (50\% mode ratio) tests with fabric-side-up are shown by the red lines in Figures 3a and 3b, respectively. The analytical results, identified by T/F and $\mathrm{T} / \mathrm{T}$ and shown in blue, are discussed in the next section. It can be observed that in both tests there was some nonlinearity in the load-displacement response before reaching the peak load. This nonlinearity starts at the initiation of delamination propagation and the fact that the load required for propagation increases after initiation is indicative of a resistance curve, or R-curve. 


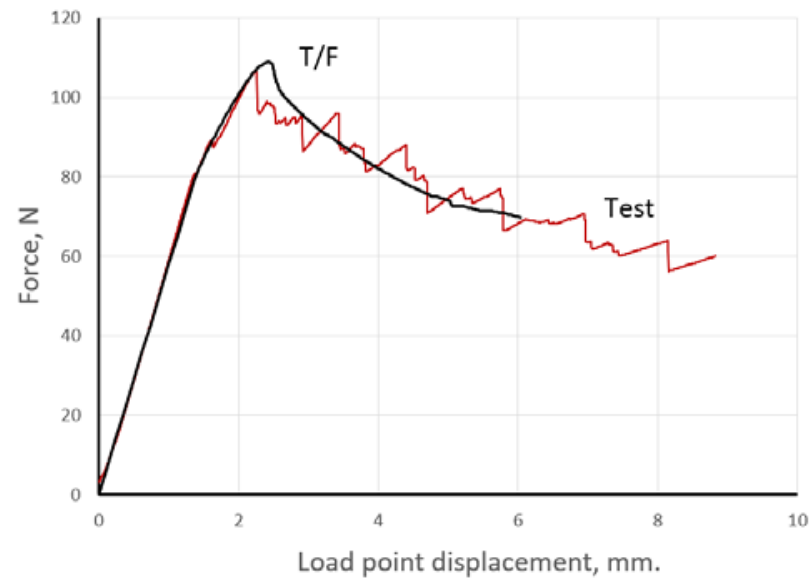

(a) Load-displacement response of DCB test

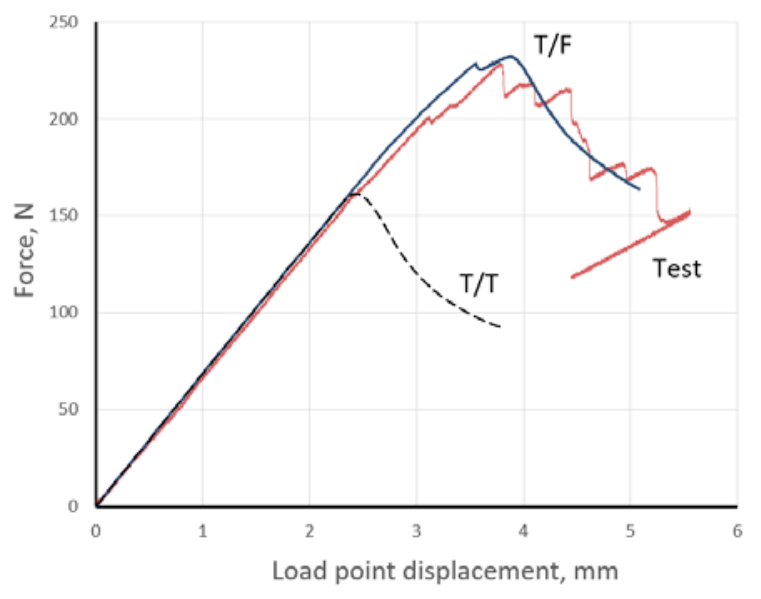

(b) Load-displacement response of MMB test

Fig. 3 Experimental and predicted load-deflection curves for DCB and MMB tests of tape/fabric interfaces (fabric-side-up, fabric-constrained).

\section{Modeling R-Curves with Superposition of Cohesive Laws}

R-curve effects are seldom taken into account in analysis, partly due to the difficulty in having to apply a value of $G_{c}$ that increases with crack propagation. Cohesive laws offer a convenient means to account for R-curves because of the direct relationship between the shape of a cohesive law and the resulting R-curve [5]. As can be expected, the shape of the cohesive law, e.g., bilinear or exponential, is inconsequential for the prediction of fracture under smallscale bridging conditions. However, under large-scale bridging conditions, where the process zone length may be large relative to other length scales in the problem, the shape of the law plays a fundamental role. When crack propagation includes different energy dissipation mechanisms that act over different length scales, the nature of these mechanisms must be accounted for in the cohesive law.

Dávila et al. [6] have shown that trilinear softening laws can represent a wide variety of R-curve responses. Trilinear laws have the benefit of being decomposable into two bilinear laws, for which specific constraints for mode mixity have been developed [7]. A comparison of the effect of a bilinear and a trilinear cohesive law on load vs. displacement predictions is illustrated in Figure 4a. Trilinear cohesive laws are necessary when there is a large difference between the $G_{c}$ for delamination initiation and for steady-state propagation.

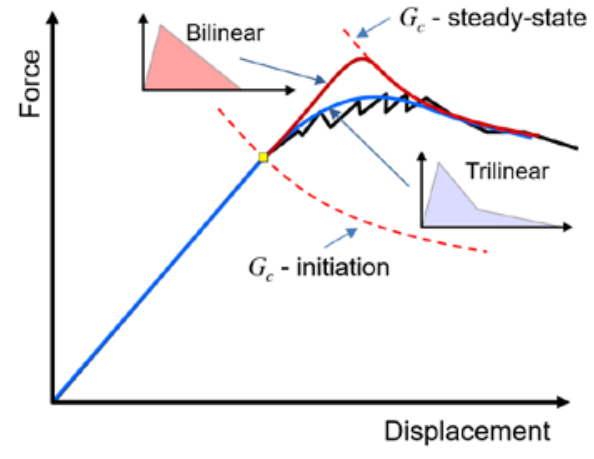

(a) Force displacement results obtained using bilinear and trilinear cohesive laws.

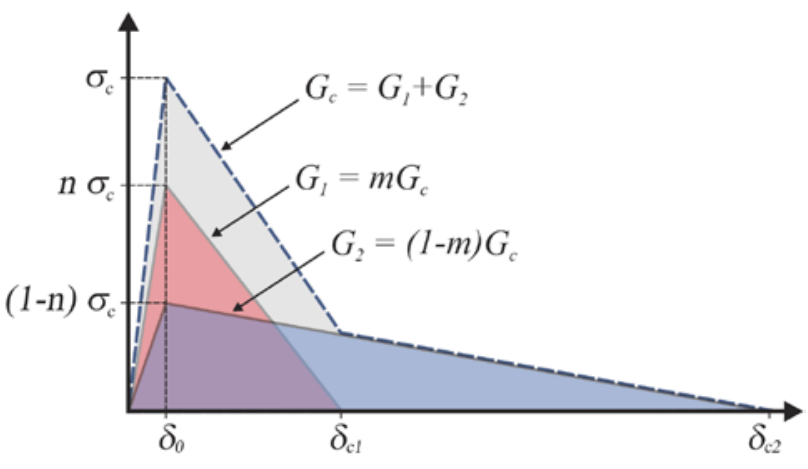

(b) Trilinear law obtained by superposition of two bilinear laws.

Fig. 4 Trilinear cohesive laws can represent a wide range of $\boldsymbol{R}$ curve effects. 
Several methods have been proposed to characterize the parameters $n$ and $m$ that describe the shape of the trilinear law (see Figure 4b). Dávila proposed a semi-empirical equation [6] relating directly a linearized fit of the R-curve to the parameters $n$ and $m$. Airoldi [8] obtained the parameters for delamination in glass-epoxy laminates by performing parametric analyses and minimizing the error between the numerical and the averaged experimental forcedisplacement responses. Bergan [9] extracted the trilinear law for intralaminar fracture by fitting an analytical form of the $J$-integral to the experimental R-curve plotted as a function of the opening displacement measured at the crack tip. Girolamo [10] used closed form solutions of the J-integral for DCB, end-notched flexure (ENF), and MMB tests to obtain the mixed mode trilinear laws describing the fracture of FM300 adhesive in a composite laminate.

In the present effort, a much simpler approach was used, which assumes that the initiation of propagation is defined by the properties of standard tape specimens, denoted as standard $\mathrm{T} / \mathrm{T}$ in Table 1 . These properties were obtained in the course of the NASA Advanced Composites Project [3,11, 12]. A second bilinear cohesive law accounts for the increase in $G_{c}$ from initiation to steady-state propagation. This second cohesive law is referred-to as bridging, because its effect is similar to the fiber bridging effect that can form in the wake of a crack tip. Two sets of bridging properties are reported in Table 1 . The values of $G_{I c}$ and $G_{I I c}$ for the fabric-constrained bridging law were obtained from DCB, MMB, and ELS tests with fabric-constrained propagation. The tensile and shear strengths of the bridging law are obtained by fitting the predicted force-displacement results with the experimental data [4]. The parameter $\eta_{B K}$ represents the parameter of the Benzeggagh-Kenane mixed-mode fracture criterion [13].

The properties for the tape-constrained bridging law were determined in a similar way based on the mode-II Rcurves reported in [2]. Although the values of $G_{I c}$ and $\sigma_{c}$ were expected to be independent of specimen orientation, these values had to be adjusted in order to fit the response of an MMB test with $20 \%$ mode mixity described in the next sections. This inconsistency, which may be due to details of the experimental procedures or non-monotonic increases in $G_{c}$ with mode mixity, needs further scrutiny.

The superposition of the standard and bridging laws is performed by duplicating the cohesive elements at the interface between the tape and the fabric, and assigning the standard properties to the original layer of cohesive elements, and the bridging properties to the duplicate layer.

Table 1. Interface properties of fabric-constrained and tape-constrained propagation in IM7/8552

\begin{tabular}{ccccccc}
\hline & $\begin{array}{c}G_{I c} \\
{\left[\mathrm{~N}-\mathrm{mm} / \mathrm{mm}^{2}\right]}\end{array}$ & $\begin{array}{c}G_{I I c} \\
{\left[\mathrm{~N}-\mathrm{mm} / \mathrm{mm}^{2}\right]}\end{array}$ & $\begin{array}{c}\sigma_{c} \\
{[\mathrm{MPa}]}\end{array}$ & $\begin{array}{c}\tau_{c} \\
{[\mathrm{MPa}]}\end{array}$ & $\begin{array}{c}K_{I} \\
{[\mathrm{MPa}]}\end{array}$ & $\eta_{B K}$ \\
\hline $\begin{array}{c}\text { Standard T/T } \\
{[3,11,12]}\end{array}$ & 0.24 & 0.74 & 90. & 98. & $5 . \mathrm{E}+5$ & 2.07 \\
\hline $\begin{array}{c}\text { Fabric-constrained } \\
\text { bridging [2, 4] }\end{array}$ & 0.35 & 2.10 & 7.0 & 21. & $3.89 E+4$ & 3. \\
\hline $\begin{array}{c}\text { Tape-constrained } \\
\text { bridging [2] }\end{array}$ & 0.08 & 0.10 & 0.5 & 1.0 & $2.78 E+3$ & 2.07 \\
\hline
\end{tabular}

The mode I penalty stiffnesses, $K_{I \_B r}$, for the bridging laws are shown in italic to indicate that they were calculated as follows:

$$
K_{I_{-} B r}=K_{I} \frac{\sigma_{c_{-} B r}}{\sigma_{c}}
$$

The constraint represented by Eq. 1 ensures that the critical displacement jump, $\delta_{0}$, of the bridging law, is the same as that of the standard law so that the sum of the standard and bridging law is trilinear, as illustrated in Fig. 4. The analyses were conducted using a user-written UMAT subroutine based on the Turon constitutive model [14]. This model, which is an updated version of the cohesive model used in Abaqus and originally developed by Camanho et al. [13], ensures thermodynamic consistency when the following constraint between the cohesive penalty stiffness in shear and the mode I penalty stiffness is enforced:

$$
K_{\text {sh }}=K_{I} \frac{G_{I c}}{G_{\text {IIc }}}\left(\frac{\tau_{c}}{\sigma_{c}}\right)^{2}
$$


The properties shown in Table 1 were applied in an Abaqus/Standard model composed of SC8R continuum shell elements. COH3D8 cohesive elements were used to tie together the two arms of the specimen. Figure 5a depicts the configuration of the MMB fixture, and Fig. 5b illustrates the model of the MMB specimen under load. In the orientation shown in Fig. 5b, the delamination is constrained by the fabric. When the specimen is placed upside-down in the MMB fixture, the delamination is constrained by the UD tape. The analyses results shown in blue in Fig. 3 indicate that the superposed cohesive laws capture quite accurately the response of the DCB and MMB specimens. In contrast, using standard T/T fracture properties of tape/tape interfaces results in a severe underprediction of the experimental results, as shown. These analyses are reported in more detail in Ref. [4].

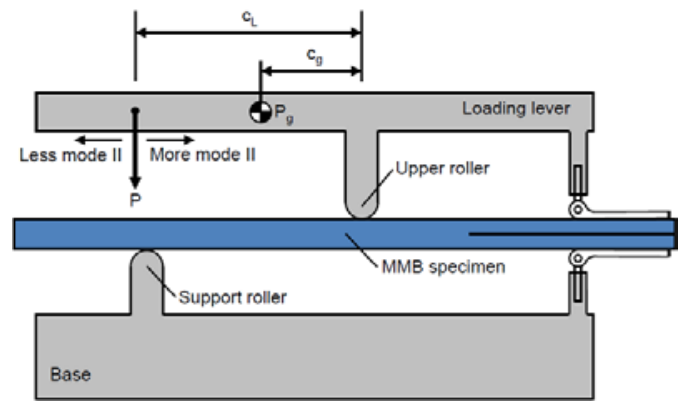

a) Configuration of MMB fixture

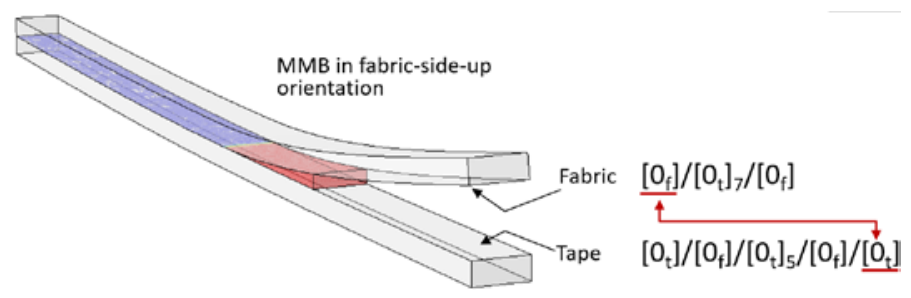

b) Model of MMB specimen in deformed configuration

Fig. 5 MMB test fixture and model of MMB Specimen

\section{Quasi-Static Response of MMB Test with Tape and Fabric Constraint}

For the present investigation, new MMB tests with a 20\% mode mixity and corresponding analyses were conducted using specimens with the same stacking sequence and configuration as described in the previous sections. To achieve a $20 \%$ mode mixity, a lever of length $C_{L}=97 \mathrm{~mm}$ was used. The average initial crack length was $26.87 \mathrm{~mm}$.

The analyses performed in the remainder of this document were performed with the two-dimensional (2D) model shown in Fig. 6. This model is composed of CPE4 plane strain elements and a layer of COH2D4 cohesive elements connecting the two arms of the specimen. This model produces satisfactory results at a lower computational cost than the three-dimensional model used in previous investigations.

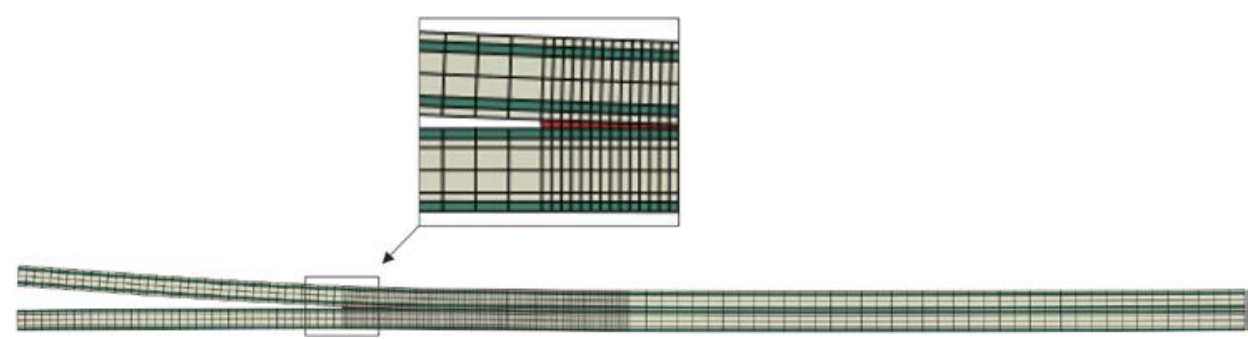

Fig. 6 2D model of MMB specimen. The green plies correspond to fabric plies, and the remaining material represents UD tape.

The experimental force-displacement results for tape-constrained propagation are shown as lines with symbols in Fig. 7a. The red curve labelled "with R-curve" represents the numerical results obtained by superposition of cohesive laws with the standard T/T and tape-constrained properties from Table 1. The blue curve corresponds to the numerical results of an analysis without using superposition, where a single bilinear cohesive law in which the critical energy release rates in modes I and II are the sum of the corresponding values for standard T/T and tape-constrained bridging. As expected, the main difference between the results with and without superposition is observed in the transition between the initiation of propagation, i.e., the point of departure from the linear response, and steady-state propagation. Two lines of constant energy release rates obtained with an analytical equation are shown for reference. The line $G_{c}$ 
$=0.263 \mathrm{~N}-\mathrm{mm} / \mathrm{mm}^{2}$ corresponds to the standard $\mathrm{T} / \mathrm{T}$ initiation for $20 \%$ mixed-mode according to the BenzeggaghKenane criterion. A second line for $G_{c}=0.3 \mathrm{~N}-\mathrm{mm} / \mathrm{mm}^{2}$ is shown for reference.

The force displacement results for fabric-constrained delamination are presented in Fig. $7 \mathrm{~b}$. The red curve corresponds to the numerical results using the superposition of the standard $\mathrm{T} / \mathrm{T}$ and the fabric-constrained bridging properties in Table 1. The curve correlates well with the steady-state range of the experimental results, although it overpredicts by about $10 \%$ the experimental results in the range immediately following the peak load. The sensitivity of the bridging law was examined by conducting a second analysis in which the value of $G_{I c}$ for the fabric-constrained bridging law was reduced from $0.35 \mathrm{~N}-\mathrm{mm} / \mathrm{mm}^{2}$ to $0.30 \mathrm{~N}-\mathrm{mm} / \mathrm{mm}^{2}$. The effect of this minor reduction in the energy release rate on the fatigue propagation rates is examined in the Section VI of this paper.

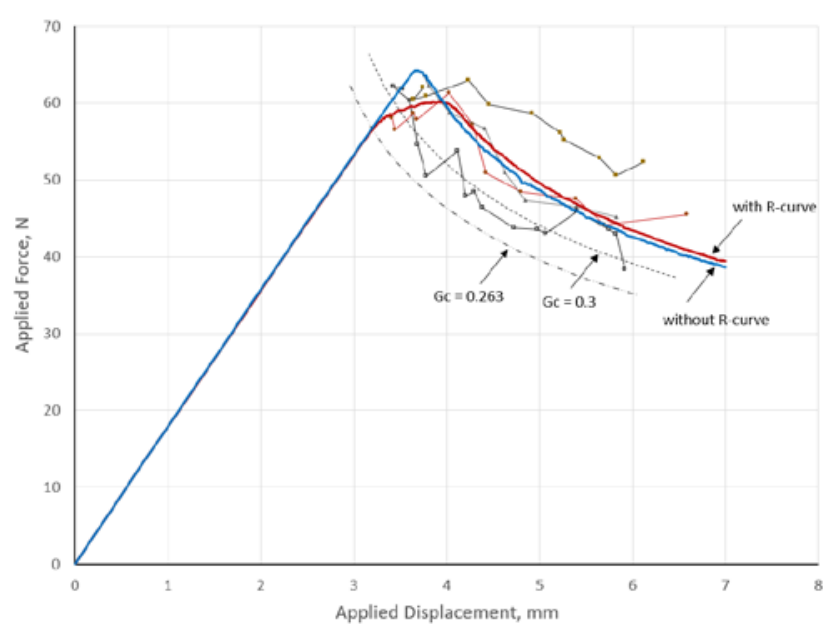

a) Tape-constrained delamination

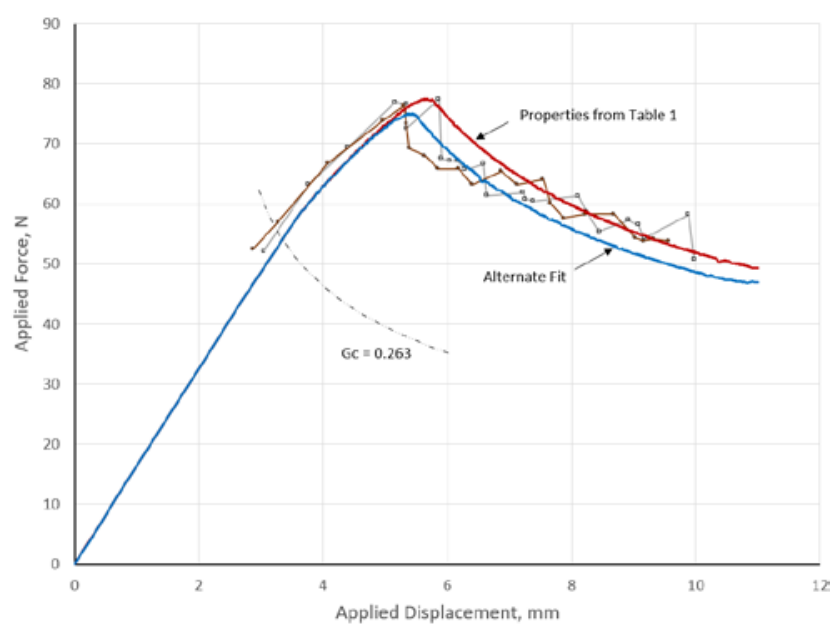

b) Fabric-constrained delamination

Fig. 7 Quasi-static force-displacement results for $20 \%$ MMB test.

\section{A Cohesive Fatigue Model Based on the Quasi-static Envelope}

The cohesive fatigue model proposed by the authors in Ref. [15] has a unique feature, which is that it relies on engineering approximations to establish the stress-life (S-N) diagrams for an interface. This determination of the S-N diagram uses the quasi-static strength, the stress ratio $R=\sigma^{\min } / \sigma^{\max }$, and the mode mixity $B$. Using the Fleck and Goodman diagram approximations [15], the model can predict crack propagation rates without any fatigue-specific material input data. The model assumes that the cohesive law is the envelope of the fatigue damage and that the evolution of fatigue damage is a function of the applied cyclic load and the damage state. The following two-parameter heuristic fatigue damage model was proposed:

$$
\frac{\mathrm{d} D}{\mathrm{~d} N}=\frac{D+\gamma}{(1-D)^{\beta}}\left(\frac{\sigma^{\max }}{\sigma_{c}}\right)^{\beta}
$$

where the damage norm $D$ is defined as:

$$
D=\frac{\lambda^{*}-\Delta^{c}}{\Delta^{f}-\Delta^{c}}
$$

and where the displacement jump, $\lambda^{*}$, and the critical displacements $\Delta^{f}$ and $\Delta^{c}$ are defined in the illustration of the cohesive envelope in Fig. 8. The stress $\sigma^{\max }$ in Eq. 3 is the maximum cyclic load, $\sigma_{c}$ is the interfacial strength, and $\gamma$ and $\beta$ are material parameters that can be obtained from the $\mathrm{S}-\mathrm{N}$ diagram as described below. 
An S-N diagram corresponding to the proposed damage model can be calculated by integrating Eq. 3 as described in [15]. The result has the form:

$$
N^{f}=\left(\frac{\sigma_{c}}{\sigma^{\max }}\right)^{\beta} \quad F\left(\frac{\sigma_{c}}{\sigma^{\max }}, \beta, \gamma\right)
$$

Where the term $F$ is approximately constant for a maximum stress $\sigma^{\max }<0.95 \sigma_{c}$. Below this stress level, Eq. 5 represents a straight line in a log-log plot of $\mathrm{N}$ versus the stress ratio, which is typical of $\mathrm{S}-\mathrm{N}$ diagrams.

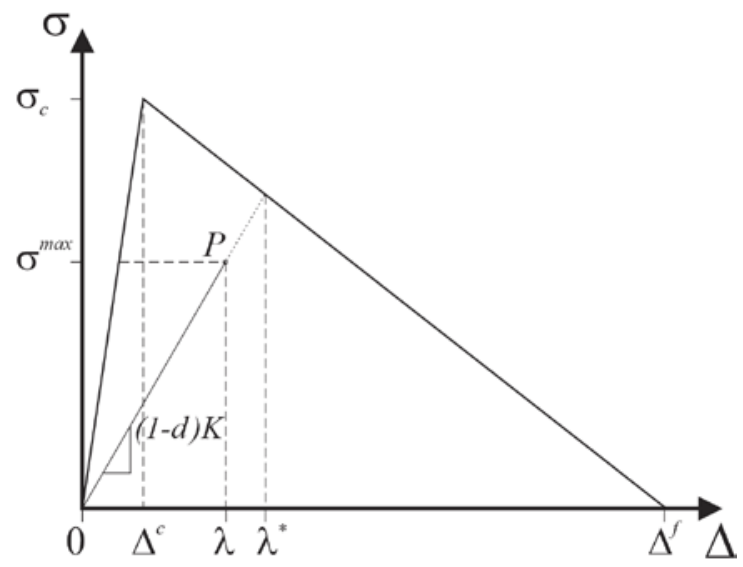

Fig. 8 Cohesive law with fatigue damage - definition of failure envelope and damage variables.

The coefficients $\beta, \gamma$ that define the damage model in Eq. 3 can be determined by fitting the results of Eq. 3 onto an experimental S-N curve or an idealized S-N curve obtained using engineering approximations. In the following analyses, the mixed-mode endurance limit, i.e., the stress $\sigma_{e}$ that causes failure in $10^{7}$ cycles is assumed to be [15]:

$$
\sigma_{e}=(1-0.42 B) \frac{\sigma_{c}}{2-R}
$$

where $B$ is the mode mixity and $R$ is the stress ratio, $\sigma^{\min } / \sigma^{\max }$.

It can be observed that the endurance limit and the corresponding S-N diagram depend on the stress ratio and the mode mixity. Even in problems such as the MMB test, where the stress ratio can be kept constant and the mode mixity is approximately constant, the S-N diagram can vary across different points in the damage zone. This variation occurs because mixed-mode damage in a flexed specimen initiates in mode II and gradually shifts to mode I as damage transitions into fracture [16]. Therefore, using experimental S-N data in mixed mode can be impractical because data for several mode mixities and a means to interpolate between the experimental values are required.

Analyses were conducted as described in [15] within a simplified cyclic loading (SCL) procedure that avoids the computational expense of having to cycle the applied load. In SCL, the maximum load is held constant. For simplicity, the frequency of cyclic loading is $1 \mathrm{~Hz}$, so that the analysis pseudo-time represents the number of cycles, as illustrated in Fig. 9. The effect of cycling on fatigue damage is represented by the stress ratio $R$ within the constitutive damage model. The analysis is conducted in two steps. The first step from time $=0$ to 1 introduces the applied load (force or displacement). No fatigue damage is allowed in the first step. During the second step, the applied load is held constant but the solution is continuously recalculated to account for the internal load redistribution that occurs with fatigue and tearing damage accumulation. 


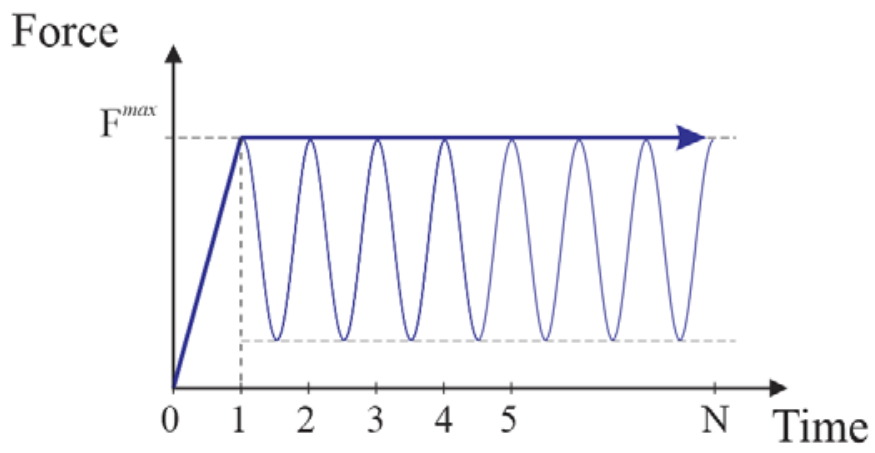

Fig. 9 SCL procedure: the load is held constant during the analysis while the effects of loading amplitude, stress ratio, cycle count, and damage accumulation are processed within the constitutive model.

\section{Fatigue Crack Propagation Rates in MMB Test with Tape and Fabric Constraint}

Fatigue tests were conducted on MMB specimens manufactured from the same batch as those used for the quasistatic tests described above. The tests were conducted under force control, with a maximum force of $F^{\max }=35.6 \mathrm{~N}$, and a stress ratio $R=0.1$. As for the quasi-static tests, tape-constrained and fabric-constrained propagation was obtained by placing the specimen in the MMB fixture in the corresponding orientation. The length of propagation as a function of cycle count was obtained from instantaneous measurements of the specimen compliance, by using a fitted cubic polynomial of the crack length as a function of the compliance. The experimental propagation rates for tape-constrained delamination are represented by the dotted lines in Fig. 10a, and the rates for fabric-constrained delamination are shown by the dotted lines in Fig. 10b.

The analysis results were processed in a manner as similar as possible to that of the experiments, so the crack length was also calculated using a cubic polynomial of the specimen compliance. Two analysis results for tapeconstrained propagation are shown in Fig. 10a. The two analyses correspond to the use of a single bilinear cohesive law (without R-curve) or using a superposed bridging law (with R-curve) as described for the quasi-static analyses in Section IV. The simulations progress from left to right because the energy release rate for a constant force increases with crack length.

It can be observed in Fig. 10a that both simulations predict an initially high propagation rate that quickly diminishes. This high rate corresponds to the formation of a fatigue process zone and the associated redistribution of stresses. According to the analysis, the simulation without R-curve (blue) reaches a minimum propagation rate after $0.6 \mathrm{~mm}$ of growth, after which the propagation rate falls within a nearly straight line that can be fitted by the typical Paris law equation. The simulation with an R-curve initially exhibits much faster propagation rates than the analysis without R-curve, until the propagation reaches steady-state and after which both simulations give similar results.

The results of simulations of the fabric-constrained propagation are shown in Fig. 10b. As for the quasi-static case, analyses were performed with the cohesive laws from Table 1, and well as an alternate fit in which the fracture toughness for the bridging law was reduced from 0.35 to $0.3 \mathrm{~N}-\mathrm{mm} / \mathrm{mm}^{2}$. It can be observed that the range of initial high propagation rates for tests and simulations are much more pronounced than for the tape-constrained propagation. This difference is attributed to the larger process zone and more pronounced bridging law required to account for the fabric-constrained propagation. According to the analyses, the minimum propagation rate is achieved after $3.2 \mathrm{~mm}$ of propagation. As in the quasi-static case, the fatigue simulation using the tabulated values fits the experimental results for longer delamination lengths better than the analyses with a reduced toughness, while the analyses using the reduced bridging toughness fit better the initial range of the experimental propagation rates.

\section{Concluding Remarks}

The differences in quasi-static and fatigue delamination for tape-constrained and fabric-constrained delamination in a tape/fabric interface were examined experimentally and numerically in a mixed-mode bending configuration with $20 \%$ mode mixity. The experimental results confirm observations made in the literature that delaminations tend to migrate towards the constraining ply that is on the compressive side of the laminate in bending. The data also indicates that the fracture toughness for a fabric-constrained delamination is almost twice that of a tape-constrained 
delamination. A cohesive model based on superposition of bilinear laws was used to account for the differences in Rcurve response for these types of surface constraints. The relationship between these quasi-static results and corresponding fatigue delamination growth rates at a fabric/tape interface was explored with a cohesive damage model that uses idealizations of stress-life diagrams used in engineering design. The fatigue model relies on the quasi-static response and does not require fatigue data for input. The fatigue model is shown to be capable of predicting the steadystate rate of delamination propagation described by the Paris law, as well as the initial transients that depend on the quasi-static R-curve effects. The analysis results help quantify the effect of fracture toughness and R-curves on the rates of delamination propagation in fatigue.

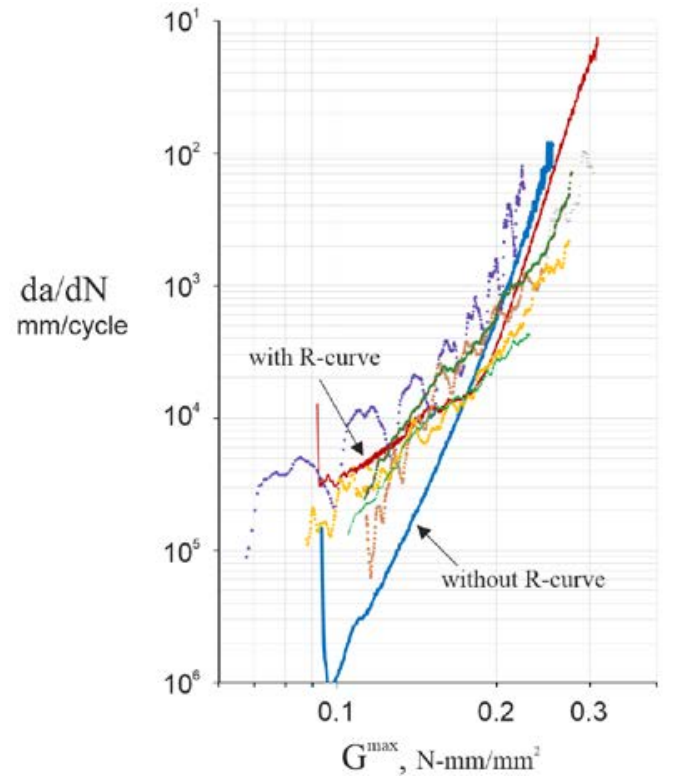

(a) Tape-constrained delamination

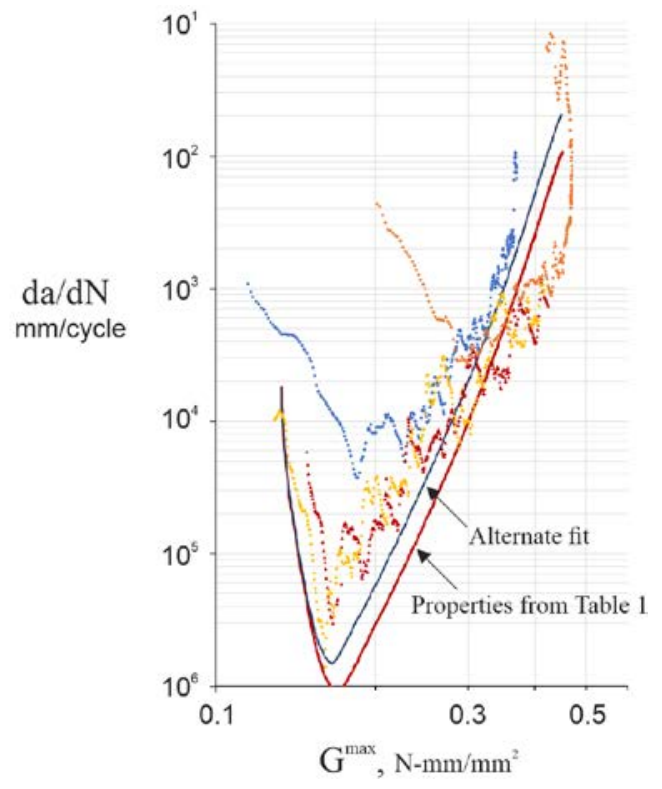

(b) Fabric-constrained delamination

Fig. 10 Delamination propagation rates in an MMB specimen at $20 \%$ mode mixity.

\section{References}

[1] Greenhalgh, E. S., Rogers, C., and Robinson, P., "Fractographic Observations on Delamination Growth and the Subsequent Migration through the Laminate," Composites Science and Technology, Vol. 69, No. 14, 2009, pp. 2345-2351.

[2] Ratcliffe, J. G., and Carvalho, N., "An Experimental Study on Effect of Containment Ply on Mode-II Delamination in 2d Woven/Unidirectional Laminates with Application to Bi-Material Interfaces," 19th International ASTM/ESIS Symposium on Fatigue and Fracture Mechanics, Denver, CO, May 15-17, 2019.

[3] Ratcliffe, J. G., and Johnston Jr, W. M., "Influence of Mixed Mode I-Mode II Loading on Fatigue Delamination Growth Characteristics of a Graphite Epoxy Tape Laminate," Proceedings of the 29th American Society for Composites Technical Conference, La Jolla, CA, 8-10 Sept. 2014.

[4] Dávila, C. G., Leone Jr, F. A., Song, K., Ratcliffe, J. G., and Rose, C. A., "Material Characterization for the Analysis of Skin/Stiffener Separation," Proceedings of the American Society for Composites, West Lafayette, IN, 22-25 Oct. 2017.

[5] Bao, G., and Suo, Z., "Remarks on Crack-Bridging Concepts," Appl Mech Rev, Vol. 45, No. 8, 1992, pp. 355366.

[6] Dávila, C. G., Rose, C. A., and Camanho, P. P., "A Procedure for Superposing Linear Cohesive Laws to Represent Multiple Damage Mechanisms in the Fracture of Composites," International Journal of Fracture, Vol. 158, No. 2, 2009, pp. 211-223. 
[7] Turon, A., Camanho, P. P., Costa, J., and Renart, J., "Accurate Simulation of Delamination Growth under MixedMode Loading Using Cohesive Elements: Definition of Interlaminar Strengths and Elastic Stiffness," Composite Structures, Vol. 92, No. 8, 2010, pp. 1857-1864.

[8] Airoldi, A., and Dávila, C. G., "Identification of Material Parameters for Modelling Delamination in the Presence of Fibre Bridging," Composite Structures, Vol. 94, No. 11, 2012, pp. 3240-3249.

[9] Bergan, A., Dávila, C., Leone, F., Awerbuch, J., and Tan, T.-M., "A Mode I Cohesive Law Characterization Procedure for through-the-Thickness Crack Propagation in Composite Laminates," Composites Part B: Engineering, Vol. 94, 2016, pp. 338-349.

[10] Girolamo, D., "Damage Progression in the Adhesive Layers of Bonded Composite Joints," Department of Aerospace Engineering, Delft University of Technology, Delft, NL, 2012.

[11] Wanthal, S., Schaefer, J., Justusson, B., Hyder, I., Engelstad, S., and Rose, C. A., "Verification and Validation Process for Progressive Damage and Failure Analysis Methods in the NASA Advanced Composites Consortium," American Society for Composites Technical Conference, Purdue University, West Lafayette, IN, 23-25 Oct. 2017.

[12] Murri, G. B., "Evaluation of Delamination Onset and Growth Characterization Methods under Mode I Fatigue Loading," Technical Report NASA/TM-2013-217966, Hampton, VA, Feb. 2013.

[13] Camanho, P. P., Dávila, C. G., and de Moura, M. F. S. F., "Numerical Simulation of Mixed-Mode Progressive Delamination in Composite Materials," Journal of Composite Materials, Vol. 37, No. 16, 2003, pp. 1415-1438.

[14] Turon, A., González, E. V., Sarrado, C., Guillamet, G., and Maimí, P., "Accurate Simulation of Delamination under Mixed-Mode Loading Using a Cohesive Model with a Mode-Dependent Penalty Stiffness," Composite Structures, Vol. 184, No. Supplement C, 2018, pp. 506-511.

[15] Dávila, C. G., "From S-N to the Paris Law with a New Mixed-Mode Cohesive Fatigue Model," Technical Report NASA-TP-2018-219838, Hampton, VA, June 2018.

[16] Sarrado, C., Turon, A., Renart, J., and Urresti, I., "Assessment of Energy Dissipation During Mixed-Mode Delamination Growth Using Cohesive Zone Models," Composites Part A: Applied Science and Manufacturing, Vol. 43, No. 11, 2012, pp. 2128-2136. 